\title{
Gravitational Energy of Kerr and Kerr Anti-de Sitter Space-Times in the Teleparallel Geometry
}

\author{
J. F. da Rocha-Neto ${ }^{a}$ and K. H. Castello-Branco ${ }^{b}$ \\ ${ }^{a}$ Instituto de Física Teórica, Universidade Estadual Paulista, \\ Rua Pamplona 145, 01405-900. São Paulo, Brazil. \\ ${ }^{b}$ Instituto de Física, Universidade de São Paulo. \\ São Paulo, Brazil. 66318, 05315-970.
}

\begin{abstract}
In the context of the Hamiltonian formulation of the teleparallel equivalent of general relativity we compute the gravitational energy of Kerr and Kerr Anti-de Sitter (Kerr-AdS) space-times. The present calculation is carried out by means of an expression for the energy of the gravitational field that naturally arises from the integral form of the constraint equations of the formalism. In each case, the energy is exactly computed for finite and arbitrary spacelike two-spheres, without any restriction on the metric parameters. In particular, we evaluate the energy at the outer event horizon of the black holes.
\end{abstract}

KEYWORDS: Classical Theories of Gravity, Black Holes $a$ E-mail: rocha@ift.unesp.br b E-mail: karlucio@fma.if.usp.br

\section{Introduction}

Teleparallel theories of gravity, whose basic entities are tetrad fields $e_{a \mu}(a$ and $\mu$ are $S O(3,1)$ and space-time indices, respectively) have been considered long time ago by Møller [1] in connection with attempts to define the energy of the gravitational field. Teleparallel theories of gravity are defined on the Weitzenböck space-time [2], which is endowed with the afinne connection $\Gamma_{\mu \nu}^{\lambda}=e^{a \lambda} \partial_{\mu} e_{a \nu}$ and where the curvature tensor, constructed out of this connection, vanishes identically. This connection defines a spacetime with an absolute parallelism or teleparallelism of vector fields [3]. In this geometrical framework the gravitational effects are due to the space-time torsion corresponding to the above mentioned connection.

Although there exists an infinity of gravity theories in this geometrical framework [4], here we will consider only the teleparallel equivalent of general relativity (TEGR) $[5,6,7,8,9,10]$. The TEGR is an alternative formulation of Einstein's general relativity 
whose corresponding tetrad fields satisfy the Einstein's equations in tetrad form. As remarked by Hehl [11], by considering Einstein's general relativity as the best available alternative theory of gravity, its teleparallel equivalent is the next best one. Therefore it is interesting to perform studies of the space-time structure as described by the TEGR.

A simple definition for the energy of the gravitational field has been established in the Hamiltonian formulation of the TEGR [7, 12] in the framework of Schwinger's time gauge condition [13]. The gravitational energy is given by an integral of a scalar density in the form of a total divergence that appears in the Hamiltonian constraint of the theory. The Hamiltonian formulation of the TEGR, with no a priori restriction on the tetrad fields, has recently been established [14]. Again, in this formulation, an expression for the gravitational energy naturally arises [15], in strict similarity with the procedure adopted in [12]. In this paper we apply this definiton of gravitational energy for the cases of Kerr and Kerr-AdS space-times. The Kerr metric is one of the most important known configurations of the gravitational field, consisting in the only vacuum rotating black hole solution of Einstein's equations [16], whereas the Kerr-AdS metric is an important AdS space. AdS spaces have received special attention in the current literature due to the AdS/CFT correspondence [17]. These spaces have also been studied earlier, as for example, in [18], where it was showed that a (large) Schwarzschild-AdS black hole is thermodynamically stable, whereas in [19] the conserved charges of AdS spaces were studied. Here we compute the gravitational energy of Kerr and Kerr-AdS black holes enclosed by an arbitrary twosphere of radius greater than or equal to their horizon radius $\left(r_{+}\right)$. The knowledge of the distribution of the gravitational energy for an arbitrary two-sphere of radius $r \geq r_{+}$is of importance in the description of other properties of the black hole space-time [20], such as an analysis of the gravitational thermodynamic not restricted to the black hole event horizon. In Ref. [20] a change in the area of black holes is related to the fluxes of energy and angular momentum caried by gravitational waves across the horizon of black holes.

Notation: space-time indices $\mu, \nu, \ldots$ and $\mathrm{SO}(3,1)$ indices $a, b, \ldots$ run from 0 to 3 . Time and space indices are indicated according to $\mu=0, i, \quad a=(0),(i)$. The tetrad field $e^{a}{ }_{\mu}$ yields the definition of the torsion tensor: $T^{a}{ }_{\mu \nu}=\partial_{\mu} e^{a}{ }_{\nu}-\partial_{\nu} e^{a}{ }_{\mu}$. The flat, Minkowski space-time metric is fixed by $\eta_{a b}=e_{a \mu} e_{b \nu} g^{\mu \nu}=(-+++)$.

\section{The Hamiltonian Formulation of the TEGR}

The Hamiltonian formulation of the TEGR developed in Ref. [14], without posing any a priori restriction on the tetrad fields, is obtained from the Lagrangian density in empty space-time, given by

$$
L(e)=-k e\left(\frac{1}{4} T^{a b c} T_{a b c}+\frac{1}{2} T^{a b c} T_{b a c}-T^{a} T_{a}\right)+2 \partial_{\mu}\left(e T^{\mu}\right),
$$

where $e=\operatorname{det}\left(e^{a}{ }_{\mu}\right), T_{a b c}=e_{b}{ }^{\mu} e_{c}{ }^{\nu} T_{a \mu \nu}, T_{b}=T_{a b}^{a}, k=\frac{1}{16 \pi G}$ and $G$ is the gravitational constant. The Lagrangian $L$ is equivalent to that of Einstein-Hilbert up to the fourdivergence. However, in the cases where boundary terms are taken into account, the surface term is necessary to leave the action integral in an appropriate form for general relativity [21]. By explicit calculations $[7,9]$ it is possible to show that the variation of 
the action integral with respect to $e_{a \mu}$ yields the Einstein's equations in tetrad form

$$
\frac{\delta L}{\delta e^{a \mu}} \equiv \frac{k}{2} e\left\{R_{a \mu}(e)-\frac{1}{2} e_{a \mu} R(e)\right\}
$$

The Hamiltonian is obtained by the prescription $L=p \dot{q}-H$ and without making any kind of projection of metric or tetrad variables to the three-dimensional spacelike hypersurface. Dispensing with surface terms, the total Hamiltonian density reads [14]

$$
H\left(e_{a i}, \Pi^{a i}\right)=e_{a 0} C^{a}+\alpha_{i k} \Gamma^{i k}+\beta_{k} \Gamma^{k} .
$$

$\left\{C^{a}, \Gamma^{k}, \Gamma^{i k}\right\}$ is a set of first class constraints, $\alpha_{i k}$ and $\beta_{k}$ are Lagrange multipliers. More details are given in Ref. [14]. The first term of the constraint $C^{a}$ is given by a total divergence in the form $C^{a}=-\partial_{k} \Pi^{a k}+Q^{a}$. As in Ref. [12] we identify this total divergence on the three-dimensional spacelike hypersurface as the energy-momentum density of the gravitational field

$$
P^{a}=-\int_{V} d^{3} x \partial_{i} \Pi^{a i}
$$

where $V$ is an arbitrary spacelike volume. It is invariant under coordinate transformations on the spacelike manifold, and transforms as a vector under the global $\mathrm{SO}(3,1)$ group. The definition above generalizes the analogous energy expression (11) of the Ref. [12] to tetrad fields that are not restricted by the time gauge condition. If the time gauge condition is imposed these expressions coincide [14].

The momenta $\Pi^{a k}$ are given by

$$
\begin{aligned}
& \Pi^{a k}=k e\left\{g^{00}\left(-g^{k j} T^{a}{ }_{0 j}-e^{a j} T^{k}{ }_{0 j}+2 e^{a k} T^{j}{ }_{0 j}\right)\right. \\
& +g^{0 k}\left(g^{0 j} T^{a}{ }_{0 j}+e^{a j} T^{0}{ }_{0 j}\right)+e^{a 0}\left(g^{0 j} T^{k}{ }_{0 j}+g^{k j} T^{0}{ }_{0 j}\right) \\
& \quad-2\left(e^{a 0} g^{0 k} T^{j}{ }_{0 j}+e^{a k} g^{0 j} T^{0}{ }_{0 j}\right)-g^{0 i} g^{k j} T^{a}{ }_{i j} \\
& \left.+e^{a i}\left(g^{0 j} T^{k}{ }_{i j}-g^{k j} T^{0}{ }_{i j}\right)-2\left(g^{0 i} e^{a k}-g^{i k} e^{a 0}\right) T^{j}{ }_{j i}\right\} .
\end{aligned}
$$

For asymptotically flat space-times, in the limit $r \rightarrow \infty$, taking the $a=(0)$ component in Eq. (4) and integrating over the whole three-dimensional spacelike hypersurface, after a straightforward calculation, we arrive at [15]

$$
P^{(0)}=\frac{1}{16 \pi G} \int_{S \rightarrow \infty} d S_{k}\left(\partial_{i} h_{i k}-\partial_{k} h_{i i}\right)=E_{A D M}
$$

which is the ADM energy [22]. The above result motivates the definition of the gravitational energy enclosed by an arbitrary volume $V$ of the three-dimensional spacelike as

$$
E_{g}=-\int_{V} d^{3} x \partial_{i}\left(\Pi^{(0) i}\right)=-\int_{S} d S_{i} \Pi^{(0) i} .
$$

Such definition has led to consistent and relevant results when applied to important configurations of the gravitational field, such as the evaluation of the irreducible mass of 
the Kerr black hole [15]. Furthermore, it gives the Bondi mass in the appropriated limit when the time gauge is imposed [15]. In fact, the definition (7) satisfies all the conditions usually required for a quasilocal gravitational energy expression.

\section{Kerr and Kerr-AdS Space-times}

In Ref. [15] the energy of the Kerr black hole was computed only to the two-sphere defined by $r=r_{+}$(outer event horizon). Differently of [15], here we compute the gravitational energy within the volume $V$ of an arbitrary two-sphere of radius $r \geq r_{+}$of a sapacelike hypersurface, without any further restriction on the metric parameters.

In terms of Boyer-Lindquist coordinates the Kerr-AdS metric is given by

$$
\begin{aligned}
d s^{2}=- & \frac{\Delta_{r}}{\rho^{2}}\left(d t-\frac{a}{\chi} \sin ^{2} \theta d \phi\right)^{2}+\frac{\rho^{2}}{\Delta_{r}} d r^{2}+\frac{\rho^{2}}{\Delta_{\theta}} d \theta^{2} \\
& +\frac{\Delta_{\theta} \sin ^{2} \theta}{\rho^{2}}\left[a d t-\frac{\left(r^{2}+a^{2}\right)}{\chi} d \phi\right]^{2},
\end{aligned}
$$

where

$$
\begin{gathered}
\Delta_{r}=\left(r^{2}+a^{2}\right)\left(1+r^{2} / l^{2}\right)-2 m r, \quad \Delta_{\theta}=1-a^{2} \cos ^{2} \theta / l^{2}, \\
\chi=1-a^{2} / l^{2}, \quad \rho^{2}=r^{2}+a^{2} \cos ^{2} \theta,
\end{gathered}
$$

and $l$ is the AdS radius, related to the cosmological constant by $\Lambda=-3 / l^{2}$. The parameters $m$ and $a$ are related to the mass and angular momentum of the black hole, respectively. In the limit $l \rightarrow \infty(\Lambda=0)$, Eq. (8) reduces to the Kerr asymptotically flat solution.

The determination of tetrads that correspond to a given metric has been carefully analysed recently in Sec. IV of [15]. A set of tetrads associated to the metric (8) is

$$
e_{a \mu}=\left(\begin{array}{cccc}
-A & 0 & 0 & 0 \\
B \sin \theta \sin \phi & C \sin \theta \cos \phi & D r \cos \theta \cos \phi & -E r \sin \theta \sin \phi \\
-B \sin \theta \cos \phi & C \sin \theta \sin \phi & D r \cos \theta \sin \phi & E r \sin \theta \cos \phi \\
0 & C \cos \theta & -D r \sin \theta & 0
\end{array}\right)
$$

where

$$
\begin{gathered}
A=\sqrt{\mathbf{A}+\frac{\mathbf{B}^{2}}{\mathbf{C}}}, \quad B=-\frac{\mathbf{B}}{\sqrt{\mathbf{C}} \sin \theta}, \quad C=\frac{\rho}{\sqrt{\Delta_{r}}}, \\
D=\frac{\rho}{r \sqrt{\Delta_{\theta}}}, \quad E=\frac{\sqrt{\mathbf{C}}}{r \sin \theta},
\end{gathered}
$$

and

$$
\begin{gathered}
\mathbf{A}=\frac{\Delta_{r}}{\rho^{2}}-\frac{a^{2} \Delta_{\theta} \sin ^{2} \theta}{\rho^{2}}, \quad \mathbf{B}=\frac{a \sin ^{2} \theta}{\rho^{2} \chi}\left(\Delta_{r}-\left(r^{2}+a^{2}\right) \Delta_{\theta}\right), \\
\mathbf{C}=\frac{\sin ^{2} \theta}{\rho^{2} \chi^{2}}\left(\Delta_{\theta}\left(r^{2}+a^{2}\right)^{2}-\Delta_{r} \sin ^{2} \theta\right) .
\end{gathered}
$$

The components of the torsion tensor obtained out of the set of tetrads above is presented in the appendix. 
For an arbitrary spacelike volume $V$ difined by a two-sphere the gravitational energy $E_{g}$ is defined relationally, in the sense that it depends on the choice of the reference space-time (see the discussion in Ref. [15]). In the Kerr case, the set of tetrads above (for $\Lambda=0$ ) amounts to choosing a unique reference space-time that is neither related by a boost transformation nor rotating with respect to the physical space-time (for a detailed discussion we refer the reader to [15]). For the Kerr-AdS case, the above tetrad field (9) is probabily the most suitable choice as well, since for $\Lambda=0$ it reduces to the tetrad field associated to the Kerr metric, as can be easily verified.

In order to obtain the energy within a spherical surface $(r=$ const $)$ of a threedimensional spacelike hypersurface of the Kerr space-time, we need to compute the component $\Pi^{(0) 1}$ of $\Pi^{a k}$, which is given in the appendix for the Kerr and Kerr-AdS cases. After that, we performe the integration (7) in the angular variables and, by means of a long, but straightforward, calculation we achieve

$$
\begin{gathered}
E_{g}=-\frac{1}{4}\left[-\sqrt{r^{2}+a^{2}}+\frac{r^{2}}{2 a} \ln \left(\frac{\sqrt{r^{2}+a^{2}}-a}{\sqrt{r^{2}+a^{2}}+a}\right)+\frac{i 2 \sqrt{\alpha}}{a} E\left(i \frac{a}{r}, \sqrt{\frac{\Delta}{\alpha}} r\right)\right. \\
\left.-\frac{i}{a} \sqrt{\frac{\Delta}{\alpha}} \partial_{r} \alpha F\left(i \frac{a}{r}, \sqrt{\frac{\Delta}{\alpha}} r\right)+\frac{i}{a} \sqrt{\frac{\alpha}{\Delta}} \partial_{r} \Delta\left[F\left(i \frac{a}{r}, \sqrt{\frac{\Delta}{\alpha}} r\right)-E\left(i \frac{a}{r}, \sqrt{\frac{\Delta}{\alpha}} r\right)\right]\right],
\end{gathered}
$$

where $i^{2}=-1$,

$$
\alpha=\left(r^{2}+a^{2}\right)^{2}-\Delta a^{2}, \quad \Delta=r^{2}+a^{2}-2 m r
$$

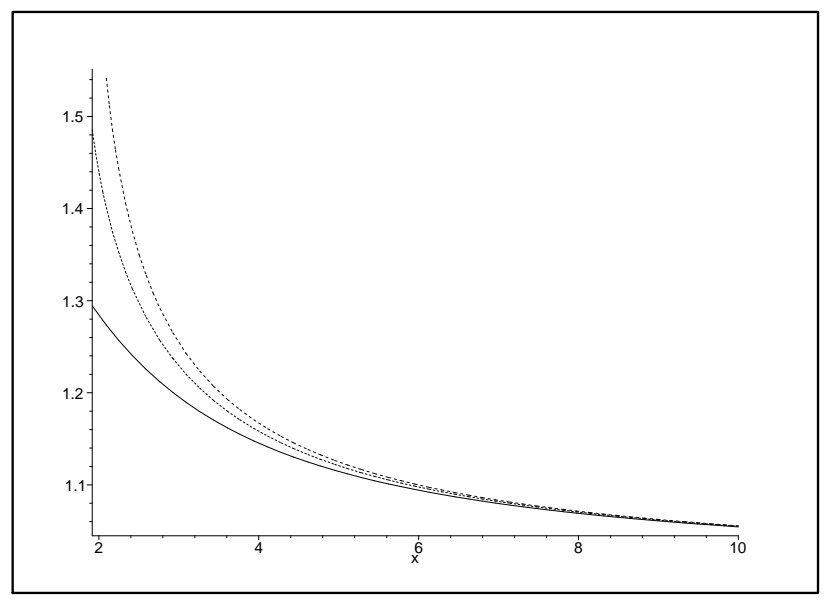

Figure 1: By using Eq. (10), here we plot $E_{g} / m$ versus $x=r / m$ for $a / m=0.4$ (doted), 0.7 (dashed), and 1.0 (solid)

and

$$
E(x, z)=\int_{0}^{x} d y \frac{\sqrt{1-z^{2} y^{2}}}{\sqrt{1-y^{2}}}
$$




$$
F(x, z)=\int_{0}^{x} d y \frac{1}{\sqrt{1-y^{2}} \sqrt{1-z^{2} y^{2}}}
$$

are the definitions of the elliptic functions $E(x, z)$

and $F(x, z)$, respectively. To arrive at the Eq. (10), the integration in the angular variables was performed, in its final part, using the Maple program. In Fig. 1 we plot the $E_{g} / m$ versus $r$ for some values of $a / m$. For large values of $r$ the energy approaches of $m$, and in the horizon the energy approaches of irreducible mass $M_{i r r}$ of the black hole.

Let us now compare the results obtained from Eq. (10) with some results in the literature. In the asymptotic limit $r \rightarrow \infty$, the gravitational energy given by (10) approaches the ADM energy $m$, and for $a=0$, we obtain the energy of the Schwarzschild black hole, in agreement with the result of Brown and York [23]. In particular, for the surface defined by $r=r_{+}$, differently of the Komar's integral [24], which gives the value $m$ [25], the above expression for the gravitational energy $E_{g}$ reduces to

$$
E_{g}\left(r_{+}\right)=m\left[\frac{\sqrt{2 p}}{4}+\frac{6 p-k^{2}}{4 k} \ln \left(\frac{\sqrt{2 p}+k}{p}\right)\right],
$$

where $p=1+\sqrt{1-k^{2}}, \quad a=k m, \quad$ and $0 \leq k \leq 1$. The result (11) is the same obtained in Ref. [15], where $E_{g}\left(r_{+}\right)$is compared with the irreducible mass $2 M_{i r r}$ of the Kerr black hole, known from the work of Christodoulou [26], as analysed in Ref. [15]. An excellent agreement was found. This agreement is a crucial test for any local or quasi-local gravitational energy expression. In [15] it is shown a small difference between (11) and $2 M_{i r r}$. However, it is argued in [28] that the Martinez conjecture [27] is not valid for large values of angular momentum.

Recently, the gravitational energy of the Kerr black hole has been analysed in the context of quasi-local energy by means of the counterterms method [28]. In [28], the gravitational energy is computed in exact, closed analytic form only at the surface defined by $r=r_{+}$. The result obtained in [28] is different from that given here by Eq. (11) (see Eq. (30) of the latter Ref.). However, in the case of slow angular momentum, the Eq. (10) can be expanded in powers of $a$ and the result obtained is strictly the same obtained by Dehghani and Mann in [28]. In Ref. [29], using pseudotensors, it is shown that the energy of the Kerr black hole is $m$ which is confined to its interior, because in that analysis the energy is independent of the surface of integration. This result is the same obtained in Ref. [25] by using the Komar's integral.

Let us now consider the gravitational energy for the Kerr-AdS space-time. For spacetimes with different topologies the appropriate gravitational action integrals require a surface term that is specific to each topology. Therefore, the correspoding Hamiltonian also acquires a surface term that is determined by the topological boundary conditions [30]. Additional terms, such as the cosmological constant, may appear in the Hamiltonian constraint. Because of this, the action integral has an extra term, as well as the Hamiltonian constraint. Therefore, in the constraint $C^{a}$ there appears an additional term given by $-2 e^{a 0} e \Lambda$.

As in the Kerr case, by means of a long but straightforward calculation, we arrive at the gravitational energy for the Kerr-AdS case contained within a surface of constant 
radius $r$. Again, we perform the integration of $\Pi^{(0) 1}$ in terms of elliptic functions. The integration is performed in exact, closed form for arbitrary two-surfaces and values of the metric parameters. The final expression for $E_{g}$ is

$$
\begin{aligned}
E_{g}=-\frac{1}{4}[ & -2 \frac{r l}{a} E\left(\frac{a}{l}, i \frac{l}{r}\right)+i 2 \frac{\sqrt{\alpha_{l}}}{\chi a} E\left(i \frac{a}{r}, \sqrt{\frac{\beta_{l}}{\alpha_{l}}} r\right)-i \frac{\sqrt{\Delta_{r}} l \partial_{r} \alpha_{l}}{a \chi \sqrt{\alpha_{l}\left(r^{2}+l^{2}\right)}} F(i \eta, \zeta) \\
& \left.+i \frac{\sqrt{\Delta_{r}} l^{3} \partial_{r} \beta_{l}}{\chi a \sqrt{\alpha_{l}\left(r^{2}+a^{2}\right)}} \times\left[-F(i \eta, \zeta)+\Pi\left(i \eta, \frac{r^{2}}{r^{2}+l^{2}}, \zeta\right)\right]\right],
\end{aligned}
$$

where

$$
\begin{gathered}
\alpha_{l}=\left(r^{2}+a^{2}\right)^{2}-\Delta_{r} a^{2}, \quad \beta_{l}=\Delta_{r}-\frac{\left(r^{2}+a^{2}\right)^{2}}{l^{2}} \\
\eta=\sqrt{\frac{r^{2}+l^{2}}{l^{2}-a^{2}}} \frac{a}{r}, \quad \zeta=\sqrt{\frac{\Delta_{r}\left(a^{2}+l^{2}\right)}{\alpha_{l}\left(r^{2}+l^{2}\right)}} r
\end{gathered}
$$

and

$$
\Pi(x, y, k)=\int_{0}^{x} d z \frac{1}{\left(1-y z^{2}\right) \sqrt{1-z^{2}} \sqrt{1-k^{2} z^{2}}}
$$

is the defination of the elliptic function $\Pi(x, y, k)$.

In the absence of angular momentum $(a=0)$ it is easy to check that (13) reduces to

$$
E_{g}=r\left(1-\sqrt{1-\frac{2 m}{r}+\frac{r^{2}}{l^{2}}}\right)
$$

which is the energy within an arbitrary spacelike surface of fixed radius $r$ in the SchwarschildAdS space-time. The result (14) has been obtained by Brown et al [31] in the context of the quasi-local energy, when the background subtraction method is used, and when the reference term is taken as $\epsilon_{0}(r)=-1 / 4 \pi r$ ( in the notation of Ref. [31]). In the limit $r \rightarrow \infty$, we find that (14) gives $E_{g} \rightarrow-\infty$. This result might be expected, since Anti-de Sitter space is a non-compact manifold with constant negative curvature.

For the especial case in which $E_{g}$ is evaluated for the surface defined by $r=r_{+}$, i.e, for the external horizon of the Kerr-AdS black hole, we find that (13) reduces to

$$
E_{g}=-\frac{1}{4}\left[-\frac{2 l r_{+}}{a} E\left(\frac{a}{l}, i \frac{l}{r_{+}}\right)+i \frac{2\left(r_{+}^{2}+a^{2}\right)}{\chi a} E\left(i \frac{a}{r_{+}}, i \frac{r_{+}}{l}\right)\right] .
$$

This result differs from that obtained in Ref. [28] in the context of the counterterms method. In the latter reference the gravitational energy inside a volume defined by the outer horizon is divergent in the limit $l \rightarrow \infty$ (see Eq. (49) in [28]). Differently of [28], in this limit, expressions (13) and (15) reduces to those of the Kerr case, given by (10) and (11), respectively. This is a prominent result of the energy expression (7), and is consistent with the fact that the metric given by Eq. (8) reduces to the Kerr metric in 
the limit $l \rightarrow \infty$. This suggests that the flat space limit of AdS space can, in fact, be defined as the limit $l \rightarrow \infty$. Such a limit has been discussed, for example, in [32] in the context of string theory. It is argued in [32] that the non-perturbative matrix theory (MTheory) in flat space can be achieved by means of the AdS-CFT correspondence. Then a holographic description of the flat space can be realized. The gravitational energy for the Kerr-AdS case, in the case of small angular momentum, can be expanded in powers of $a$ and the result obtained is very similar to the corresponding one obtained in [28].

In Ref. [33] conserved charges for spacetimes with local Anti-de Sitter asymptotic geometry is proposed. The conserved charges for Ker-AdS spacetime in Ref. [33] are calculated considering as a reference spacetime that is not the Minkowski spacetime, but the Anti-de Sitter spacetime. So in this case in the absence of angular momentum and mass the charges are vanishes. On the other hand in our paper the background spacetime is the Minkowski spacetime that is characterized by the total absence of energy and for which our energy expression vanishes. The result presented in [33] in the limite $l \rightarrow \infty$, differently from that presented here for the energy expression, reduces to $m$ that is the same obtained with Komar's charge. Moreover, as remarked by Faddeev [34], an expression for gravitational energy must vanish only in the absence of matter and gravitational field; consequently when the metric is that of the Minkowski spacetime.

\section{Concluding remarks}

In this paper we have computed the gravitational energy in the space-times of Kerr and Kerr-AdS black holes. In each case the gravitational energy has been evaluated exactly, in analytic closed form for an arbitrary two-sphere of radius $r \geq r_{+}$, without any further restriction on the metric parameters. This is the major result of this work and constitutes an advantage of the TEGR approach for the description of the gravitational field energy as compared to those based on the Hilbert-Einstein action integral, where the energy has been calculated exactly only in particular cases of the Kerr and Kerr-AdS space-times (see, for example, [28]). Our results for the gravitational energy of the Kerr-AdS black hole reduce naturally to those of Kerr in the limit $l \rightarrow \infty(\Lambda=0)$. This is a consistent

result of the gravitational energy expression (7) and indicates that it is possible in fact to define the flat space limit of the AdS space as $l \rightarrow \infty$.

Finally, in view of the above results we expect that the discussed expression for the gravitational field energy to be useful in the study of the thermodynamics of self-graviting systems, particularly in AdS space-times.

\section{Appendix: Calculation of torsion and momenta}

Now we present the nonvanish components of the torsion tensor related to the set of tetrads given in Eq. (9). They are:

$$
\begin{gathered}
T_{(0) 01}=\partial_{r} A \\
T_{(0) 02}=\partial_{\theta} A, \\
T_{(1) 01}=\sin \phi \sin \theta \partial_{r}(B / \sqrt{C}),
\end{gathered}
$$




$$
\begin{gathered}
T_{(1) 02}=\sin \phi \partial_{\theta}(B / \sqrt{C}), \\
T_{(1) 03}=\cos \phi(B / \sqrt{C}), \\
T_{(1) 12}=\cos \theta \cos \phi \partial_{r}\left(\rho / \sqrt{\Delta_{\theta}}\right)-\cos \phi \partial_{\theta}\left(\sin \theta \rho / \sqrt{\Delta_{r}}\right) \\
T_{(1) 13}=\sin \theta \sin \phi\left(\rho / \sqrt{\Delta_{r}}\right)-\sin \phi \partial_{r} \sqrt{C}, \\
T_{(1) 23}=\cos \theta \sin \phi\left(\rho / \sqrt{\Delta_{\theta}}\right)-\sin \phi \partial_{\theta} \sqrt{C}, \\
T_{(2) 01}=-\cos \phi \partial_{r}(B / \sqrt{C}), \\
T_{(2) 02}=-\cos \phi \partial_{\theta}(B / \sqrt{C}), \\
T_{(2) 03}=\sin \theta(B / \sqrt{C}), \\
T_{(2) 12}=\cos \theta \sin \phi \partial_{r}\left(\rho / \sqrt{\Delta_{\theta}}\right)-\sin \phi \partial_{\theta}\left(\sin \theta \rho / \sqrt{\Delta_{r}}\right), \\
T_{(2) 13}=\cos \phi \partial_{r} \sqrt{C}-\sin \theta \cos \phi\left(\rho / \sqrt{\Delta_{r}},\right. \\
T_{(2) 23}=\cos \theta \partial_{\theta} \sqrt{C}-\cos \theta \cos \phi\left(\rho / \sqrt{\Delta_{\theta}}\right), \\
T_{(3) 12}=-\sin \theta \partial_{r}\left(\rho / \sqrt{\Delta_{\theta}}\right)-\partial_{\theta}\left(\cos \theta \rho / \sqrt{\Delta_{r}}\right) .
\end{gathered}
$$

From the above we can use Eq. (5) to show that $\Pi^{(0) 1}$ for the Kerr-AdS case is given by

$$
\begin{aligned}
\Pi^{(0) 1} & =-2 k\left[\frac{\rho \sin \theta}{\sqrt{\Delta_{\theta}}}+\frac{\sin \theta}{\chi \rho}\left(\Delta_{\theta}\left(r^{2}+a^{2}\right)^{2}-\Delta_{r} a^{2} \sin ^{2} \theta\right)^{1 / 2}\right. \\
& \left.-\frac{\sqrt{\Delta_{r}} \sin \theta}{\chi \rho \sqrt{\Delta_{\theta}}} \partial_{r}\left(\Delta_{\theta}\left(r^{2}+a^{2}\right)^{2}-\Delta_{r} a^{2} \sin ^{2} \theta\right)^{1 / 2}\right] .
\end{aligned}
$$

For $l \rightarrow \infty(\Lambda=0)$, we obtain $\Pi^{(0) 1}$ corresponding to the Kerr case

$$
\Pi^{(0) 1}=-2 k \sin \theta\left(\rho+\frac{1}{\rho} \sqrt{\alpha+\beta \cos ^{2} \theta}-\frac{\sqrt{\Delta}}{\rho} \partial_{r} \sqrt{\alpha+\beta \cos ^{2} \theta}\right) .
$$




\section{Acknowledgements}

This work was supported by FAPESP-Brazil. The authors are grateful to J. W. Maluf for the critical reading of the manuscript and comments. J. F. R-N would like to thank J. G. Pereira for the hospitality at the Instituto de Física Teórica-IFT/UNESP-Brazil.

\section{References}

[1] C. Møller, Tetrad Fields and Conservation Laws in General Relativity, Proceedings of the International School of Physics "Enrico Fermi", edited by C. Møller (Academic Press, London, 1962); Conservation Laws in the Tetrad Theory of Gravitation, Proceedings of the Conference on Theory of Gravitation, Warszawa and Jablonna 1962 (Gauthier-Villars, Paris, and PWN-Polish Scientific Publishers, Warszawa, 1964) (NORDITA Publications No. 136).

[2] R. Weitzenböck, Invarianten Theorie (Nordhoff, Groningen, 1923).

[3] J. A. Schouten, Ricci Calculus, 2nd ed. (Springer-Verlag, London, 1954), p. 142.

[4] K. Hayashi, Phys. Lett. 69B, 441 (1977); K. Hayashi and T. Shirafuji. Phys. Rev. D19, 3524 (1979); Phys. Rev. D24, 3312 (1981).

[5] F. W. Hehl, in Proceedings of the 6th School of Cosmology and Gravitation on Spin, Torsion, Rotation and Supergravity, Erice, 1979, edited by P. G. Bergmann and V. de Sabbata (Plenum, New York, 1980); F. W. Hehl, J. D. McCrea, E. W. Mielke and Y. Ne'eman, Phys. Rep. 258, 1 (1995).

[6] J. M. Nester, Int. J. Mod. Phys. A 4, 1755 (1989); J. Math. Phys. 33, 910 (1992).

[7] J. W. Maluf, J. Math. Phys. 35, 335 (1994).

[8] V. C. de Andrade and J. G. Pereira, Phys. Rev. D56, 4689 (1997).

[9] M. Blagojevic' and I. A. Nikolic', Phys. Rev. D 62, 024021 (2000).

[10] E. W. Mielke, Ann. of Phys. 219, 78 (1992).

E. W. Mielke, Phys. Lett. A 251, 349 (1999).

[11] F. W. Hehl, in Proceedings of the 8th Marcel Grossman Meeting, Jerusalem, 1997, edited by Tsvi Piran (World Scientific, Singapore, 1998).

[12] J. W. Maluf, J. Math. Phys. 36, 4242 (1995).

[13] J. Schwinger, Phys. Rev. 130, 1253 (1963).

[14] J. W. Maluf and J. F. da Rocha-Neto, Phys. Rev. D 64, 084014 (2001). 
[15] J. W. Maluf, J. F. da Rocha-Neto, T. M. Toríbio and K. H. Castello-Branco, Phys. Rev. D 65, 124001 (2002).

[16] J. Bicak, Einstein Field Equations and Their Physical Implications (Selected Essays in Honour of Juergen Ehlers), B. Schmidt (ed.), Lecture Notes in Physics, Vol. 540. Springer-Verlag (2000).

[17] J. Maldacena, Adv. Theor. Math. Phys. 2, 231 (1998).

[18] S. Hawking and D. Page, Comm. Math. Phys. 87, 577 (1983).

[19] M. Hennaux and C. Teiltelboim, Comm. Math. Phys. 98, 391 (1985).

[20] A. Ashtekar and Badri Krishnan gr-qc/0207080.

[21] R. M. Wald, General Relativity, The University of Chicago Press, Chicago (1984).

[22] R. Arnowitt, S. Deser and C. W. Misner, in Gravitation: an Introduction to Current Research, edited by L. Witten (Wiley, New York, 1962).

[23] J. D. Brown and J. W. York Jr., Phys. Rev. D 47, 1407 (1993).

[24] A. Komar, Phys. Rev. 113, 934 (1959).

[25] G. Bergqvist, Class. Quantum Grav. 9, 1753 (1992).

[26] D. Christodoulou, Phys. Rev. Lett. 25, 1596 (1970).

[27] E. Martinez, Phys. Rev. D 50, 4920 (1994).

[28] M. H. Dehghani and R. B. Mann, Phys. Rev. D 64, 044003 (2001).

[29] J. M. Aguirregabira, A. Chamoro and K. S. Virbhadra, Gen. Rel. Grav. 28, 1393 (1996).

[30] S. W. Hawking and G. T. Horowitz, Class. Quant. Grav. 13, 1487 (1996).

[31] J. D. Brown, J. Creighton and R. B. Mann, Phys. Rev. D 50, 6394 (1994).

[32] L. Susskind, hep-th/9901079.

[33] R. Aros, M. Contreras, R. Olea, R. Troncoso and J. Zanelli, Phys. Rev. D 84, 1647 (2000).

[34] L. D. Faddeev, Sov. Phys. Usp. 25(3), 130 (1982). 